\title{
Malformaciones congénitas en hijos de madres con diabetes gestacional
}

\author{
Julio Nazer Herrera1 ${ }^{1}$, Moira G arcía Huidobro², \\ Lucía Cifuentes 0 valle ${ }^{3}$.
}

\section{Congenital malformations among offspring of diabetic women}

Background: The risk of congenital malformations is two to three times higher among women with diabetes mellitus before pregnancy. Gestacional diabetes is emerging as a risk factor for malformations. Aim: To study the rate and type of congenital malformations among offspring of women with pre-gestational or gestational diabetes. Material and methods: A search in the databases of the Collaborative Latin American Study for Congenital Malformations and of the Neonatology Unity of The University of Chile Clinical Hospital. All births from January 1998 through June 2004 were investigated. Results: In the study period, 13,965 births occurred, of which $0.7 \%$ were stillbirths and $8.1 \%$ had malformations. A total of 295 women (2.1\%) had diabetes (gestational diabetes in $1.8 \%$ and pre gestational diabetes in $0.3 \%$ ). The prevalence of congenital malformations was $14 \%$ and $18.3 \%$ among offspring of mothers with pre-gestational and gestational diabetes, respectively $(\mathrm{p}=\mathrm{NS})$. The prevalence of congenital malformations among offspring of non diabetic women was 7.9\% ( $p<0.001$ with respect to diabetic women). Major malformations were found in $83 \%$ and $70 \%$ of malformed offspring of women with pre-gestational and gestational diabetes, respectively. Conclusions: Pregnant women with pre-existing or gestational diabetes have a higher risk of giving birth a child with malformations (Rev Méd Chile 2005; 133: 547-54).

(Key Words: Abnormalities; Congenital, hereditary, and neonatal diseases and abnormalities; Diabetes, gestational)

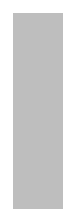

\footnotetext{
Recibido el 21 de octubre, 2004. Aceptado en versión corregida el 28 de marzo, 2005.

${ }^{1}$ Unidad de Neonatología, Departamento de Obstetricia y Ginecología, Hospital Clínico Universidad de Chile, Facultad de Medicina.

${ }^{2}$ Becaria de Anestesiología, Universidad de Valparaíso.

${ }^{3}$ Programa de Genética Humana, Instituto de Ciencias Biomédicas, Facultad de Medicina, Universidad de Chile.
}

Correspondencia a: Dr Julio Nazer H. Troncos Viejos 1899, La Reina, Santiago. Fono: 277 4106. E-mail: julionazer@mi-mail.cl 
$\mathrm{H}$ ay dos hechos demostrados en la literatura médica que se refieren a diabetes y embarazo, que $0,2 \%$ a $0,3 \%$ de los embarazos tienen diabetes mellitus (DM) preexistente, mientras que $1 \%$ a $5 \%$ de los embarazos se complican con diabetes gestacional (DG). También se ha demostrado que la prevalencia de defectos congénitos al nacimiento, en madres diabéticas, se estima entre $8 \%$ y $10 \%$, mientras que en la población general esta cifra es alrededor de 3\% ${ }^{1}$. El ECLAMC (Estudio Colaborativo Latino Americano de Malformaciones Congénitas), en una revisión de más de 4 millones de nacimientos entre 1967 y 1997, encontró que el antecedente de diabetes materna, de cualquier tipo, en el primer trimestre del embarazo de mujeres que habían tenido un hijo malformado, estaba en 6 por 100 de ellos ${ }^{2}$.

Los factores teratogénicos relacionados con la embriopatía diabética son muchos: insulina, hiperglicemia, cuerpos cetónicos, alteraciones de la glicólisis, déficit de ácido araquidónico, inhibición de la somatomedina ${ }^{3}$.

Los riesgos que presentan los hijos de madres diabéticas al nacimiento son varios, destacando alteraciones del crecimiento fetal intrauterino (40\%), hipoglicemia (20\%), prematurez (15\%), asfixia (15\%), enfermedad de membrana hialina (15\%), malformaciones congénitas (MC) (5 a 12\%) y trastornos metabólicos como hipocalcemia, hipomagnesemia e hiperbilirrubinemia ${ }^{3}$.

Las malformaciones mayores siguen siendo la gran causa de mortalidad y de morbilidad grave en hijos de mujeres con diabetes pregestacional de tipo 1 y 2, además de altas tasas de abortos espontáneos en el período embrionario ${ }^{4}$.

Se ha avanzado mucho en el conocimiento de la diabetes y del manejo de la mujer diabética embarazada. Sin embargo, los resultados para evitar los problemas del hijo parecen no haber disminuido.

Se define diabetes gestacional como cualquier grado de intolerancia a la glucosa, que haya comenzado o se haya reconocido por primera vez durante el embarazo, independientemente de que persistan o no después de la gestación. Las mujeres con diabetes gestacional corren mayor riesgo de sufrir diabetes, generalmente tipo 2, después del embarazo. La obesidad y otros factores promotores de la resistencia a la insulina aumentan el riesgo de diabetes de tipo 2. Se ha visto también, que los hijos de madres con diabetes gestacional corren mayor riesgo de obesidad y diabetes en la adolescencia y en la edad adulta ${ }^{4}$.

En la actualidad, la literatura médica ha sostenido el concepto de que los embarazos complicados con diabetes gestacional tienen el mismo riesgo para malformaciones fetales que las mujeres no diabéticas. Schaefer et $\mathrm{al}^{4}$ han comunicado una incidencia aumentada de anomalías, habitualmente asociadas con diabetes tipo 1, en mujeres ya sea diabéticas gestacionales o diabetes tipo 2 . Las anomalías estaban asociadas con hiperglicemias en ayuno y valores elevados de hemoglobina glicosilada y puede corresponder a mujeres con diabetes mellitus (DM) pregestacionales tipo 2 no diagnosticadas ${ }^{4}$.

La asociación de DM materna con anomalías congénitas es bien conocida, especialmente las mayores de los sistemas cardiovascular, nervioso central (SNC), genitourinario y esquelético. Los estudios también muestran que los hijos de mujeres con DG, especialmente aquellas con hiperglicemia en ayunas, tienden a tener tasas más altas de $\mathrm{MC}^{5}$

Los hijos de madres con DG o pregestacional, asociado a una hiperglicemia en ayunas (mayor de $105 \mathrm{mg} / \mathrm{dl}$ ), tienen un riesgo tres a cuatro veces mayor de malformaciones. En la DG con buen control metabólico la tasa de MC no difiere de la de la población no diabética ${ }^{6}$.

El diagnóstico de DG ha sido asociado a un aumento en la prevalencia de MC, sin embargo, las pacientes con DG pueden representar un grupo de diabéticas tipo 2 que ha presentado hiperglicemia durante la organogénesis. Este riesgo puede ser sustancialmente disminuido con un control de glicemia en ayunas preconcepcional y durante el embarazo ${ }^{6}$

\section{OBjetivos}

El objetivo principal de este trabajo es determinar la prevalencia, al nacimiento, de malformaciones congénitas en los hijos de las madres diabéticas en forma global, y separado por tipo de diabetes (DG y DM preconcepcional) y compararlas con la tasa de malformaciones congénitas de los hijos de madres no diabéticas. Determinar el tipo de malfor- 
maciones que presentan cada una de ellas, tanto las anomalías mayores como menores. Estudiar cómo se comportan algunos factores epidemiológicos, como edad materna, peso de nacimiento, sexo, antecedentes de otros malformados en la familia, mortinatos y de abortos previos.

\section{Pacientes y MÉtodo}

La maternidad del Hospital Clínico de la Universidad de Chile (HCUCH) es integrante del ECLAMC desde 1969, y lleva un registro de todas las malformaciones congénitas de los recién nacidos (RN), tanto nacidos vivos como mortinatos de peso mayor de $500 \mathrm{gr}$, los que se ingresan a una base de datos. Como control, se toma al RN vivo, del mismo sexo, que nace a continuación y que no presenta malformaciones. Se estudió el período comprendido entre el 1 de enero de 1998 y el 30 de junio de 2004.

Se estudiaron los antecedentes de las madres diabéticas gestacionales y pregestacionales y, para los efectos de este trabajo, como grupo control se tuvo que confeccionar una muestra aleatoria de madres no diabéticas, debido a que entre los antecedentes de los RN controles sanos del registro ECLAMC, había madres diabéticas gestacionales y pregestacionales. Se eligió a los dos primeros RN malformados, hijos de madres no diabéticas, de los meses de enero y los dos primeros RN no malformados, hijos de madres no diabéticas de los meses de febrero a diciembre de cada año en estudio. Así, se formó un grupo de $146 \mathrm{RN}, 14$ malformados y 132 no malformados hijos de madres no diabéticas.
La comparación de variables cualitativas (frecuencia de malformaciones y antecedente de abortos espontáneos previos) entre los grupos, se hizo con prueba de $\chi^{2}$. La comparación de variables cuantitativas (peso de nacimiento y edad materna) se hizo mediante análisis de varianza a un factor y a dos factores, con comparaciones múltiples en base a prueba de Tukey. Para todas las pruebas estadísticas se usó un nivel de significación de $\alpha=0,05$.

\section{Resultados}

En el período estudiado, ocurrieron 13.965 nacimientos consecutivos, de los que 13.872 fueron nacidos vivos (NV) y 93 mortinatos (NM), lo que representa una mortinatalidad de $0,66 \%$. Se encontró $1.138 \mathrm{RN}$ que presentaban una o más anomalías congénitas (8,14\%, 1.138/13.965), $8,04 \%$ en NV (1.116/13.872) y $23,65 \%$ en mortinatos $(22 / 93)$.

En 295 embarazadas (2,1\%) se diagnosticó algún tipo de diabetes, de las que 252 presentaron DG $(1,8 \%)$ y $43(0,3 \%)$ DM pregestacional. En este grupo de mujeres con algún tipo de diabetes, hubo $52(17,6 \% ; 52 / 295)$ que tuvieron hijos que presentaban una o más malformaciones congénitas. De ellas 46 eran casos de DG $(18,3 \%)$ y 6 de DM pregestacional (14\%). La tasa de frecuencia de $\mathrm{RN}$ malformados entre las mujeres no diabéticas fue de $7,9 \%$ (1.083/13.670) Tabla 1.

Al comparar el porcentaje de $\mathrm{RN}$ malformados entre madres con DG o con DM, se comprobó que no existió diferencia significativa entre ellas $\left(\chi^{2}=0,47 ; p=0,49\right)$.

Tabla 1. Prevalencia al nacimiento de malformaciones congénitas en hijos de madres diabéticas, gestacional y mellitus, y madres no diabéticas. M aternidad H ospital C línico U niversidad de C hile. 1998-2004

\begin{tabular}{|lcccc|}
\hline Recién nacido & Madres & Madres & Madres & Total \\
& No diabéticas & Diabetes gestacional & Diabetes mellitus & \\
\hline Sano & $12.587(92,1 \%)$ & $206(81,7 \%)$ & $37(86,0 \%)$ & 12.830 \\
Malformado & $1.083(7,9 \%)$ & $46(18,3 \%)$ & $6(14,0 \%)$ & $1.135(8,12 \%)$ \\
Total & 13.670 & 252 & 43 & 13.965 \\
\hline
\end{tabular}

$\chi^{2}=37,3$ con dos grados de libertad; $\mathrm{p}<0,00001$. 
Por el contrario, la prevalencia al nacimiento de recién nacidos portadores de malformaciones congénitas, fue significativamente diferente tanto en las madres DG como en las DM al compararlas con las madres no diabéticas ( $\mathrm{p}<0,00001$ ).

De los 6 RN hijos de madres con DM encontramos 5 malformaciones mayores (83,3\%): 3 con cardiopatías congénitas (2 casos de miocardiopatía, una de ellas con ductus arterioso amplio y una comunicación interventricular), 1 anencefalia, 1 imperforación anal y 1 nevus pigmentado. Entre los $46 \mathrm{RN}$ hijos de madres con diabetes gestacional, encontramos 32 pacientes $(69,6 \%)$ con una o más anomalías mayores: 8 pacientes con cardiopatías congénitas (algunos con más de una), 8 malformaciones esqueléticas ( 3 pie bot, 2 displasias de caderas, 2 casos de ausencia de dedos y 1 pulgar trifalángico), 3 con malformaciones múltiples, no sindrómicas (1 caso de regresión caudal, 1 caso de secuencia de Pierre Robin, 1 sin diagnóstico patogénico), 3 con síndrome de Down, 2 onfaloceles, 2 fisuras labiopalatinas, 2 casos de malformaciones renales, 1 atresia de duodeno, 1 hidrocefalia y 1 quiste branquial y 14 $\mathrm{RN}$ con defectos menores como angiomas, nevi pigmentados, fístulas auris, papilomas preauriculares, criptorquidia o politelias (Tabla 2). Entre los recién nacidos malformados hijos de madres no diabéticas encontramos 10 (58\%) con malformaciones mayores y $7(41,2 \%)$ con malformaciones menores (algunos tenían más de una malformación).

Cuando comparamos el antecedente de abortos espontáneos previos entre los tres grupos de madres, no se encontró diferencia significativa entre ellas $\left(\chi^{2}=3,92\right.$, con dos grados de libertad; $\mathrm{p}=0,14)$.

En la Tabla 3, se compara el peso de nacimiento de los recién nacidos no malformados y recién nacidos malformados hijos de las madres no diabéticas, con los hijos no malformados y malformados de madres diabéticas, gestacionales y pregestacionales. Los datos de peso de nacimiento se obtuvieron sólo en aquellas madres con ficha

Tabla 2. M alformaciones encontradas en los hijos de madres con diabetes gestacional

\begin{tabular}{|lrr|}
\hline Diagnóstico & $\mathrm{n}$ & $\%$ \\
\hline Cardiopatías congénitas & 8 & 17,4 \\
Malformaciones esqueléticas & 7 & 15,2 \\
Malformaciones múltiples & 4 & 8,7 \\
Síndrome de Down & 3 & 6,5 \\
Malformaciones renales & 2 & 4,3 \\
Onfaloceles & 2 & 4,3 \\
Fisuras labiopalatinas & 2 & 4,3 \\
Atresia de duodeno & 1 & 2,2 \\
Síndrome de Pierre Robin & 1 & 2,2 \\
Quiste branquial & 1 & 2,2 \\
Hidrocefalia & 1 & 2,2 \\
Total malformaciones mayores & 32 & 69,6 \\
Total malformaciones menores & 25 & $54,3^{*}$ \\
\hline
\end{tabular}

"Algunos RN presentaron más de una malformación.

Tabla 3. Peso de nacimiento (en gramos), (promedio, desviación estándar y número de casos), según la condición del RN (malformado o no) y según la condición de la madre (diabetes gestacional (D G ), diabetes pregestacional (D M ) o no diabética (N D)

\begin{tabular}{|lccccccccc|}
\hline $\begin{array}{l}\text { Condición } \\
\text { de la madre }\end{array}$ & \multicolumn{1}{c}{$\begin{array}{c}\text { Sano } \\
\text { Desv. St. }\end{array}$} & $\mathrm{n}$ & $\overline{\mathrm{x}}$ & $\begin{array}{c}\text { Malformado } \\
\text { Desv. St }\end{array}$ & $\mathrm{n}$ & $\overline{\mathrm{x}}$ & $\begin{array}{c}\text { Total } \\
\text { Desv. St. }\end{array}$ & $\mathrm{n}$ \\
\hline ND & 3407,2 & 576,0 & 132 & 3086,1 & 583,3 & 14 & 3378,4 & 582,1 & 146 \\
DG & 3606,1 & 602,5 & 52 & 3286,5 & 709,4 & 46 & 3456,1 & 679,9 & 98 \\
DM & 4013,1 & 342,0 & 18 & 3394,2 & 539,2 & 6 & 3858,3 & 473,9 & 24 \\
Total & 3597,4 & 591,5 & 202 & 3253,8 & 668,9 & 66 & 3447,2 & 619,0 & 268 \\
\hline
\end{tabular}


ECLAMC, y que son 98 con DG y 24 DM, las cuales consideramos representativas del grupo total de madres diabéticas, ya que constituyen una muestra aleatoria de éstas. El análisis de varianza para peso de nacimiento (Tabla 4), demostró que tanto la condición de recién nacido, como la presencia de diabetes en la madre tuvieron un efecto estadísticamente significativo sobre éste, $p$ $<0,0001$ en el caso de presencia de malformación en el RN y $\mathrm{p}=0,014$ en el caso de diabetes materna, ambos factores con un efecto inverso, es decir, la condición de madre diabética se asoció a mayor peso de nacimiento del hijo y la condición de RN malformado se asoció a menor peso de nacimiento, no existiendo interacción significativa entre ambos factores $(\mathrm{p}=0,602)$. En el caso de la diabetes materna se demostró, con la prueba de
Tukey, que el grupo de niños que difiere fue el de hijos de madres con diabetes mellitus pregestacional ( $p<0,05)$, no existiendo diferencias significativas de peso de nacimiento entre hijos de madres con diabetes gestacional y madres no diabéticas.

En la Tabla 5 se presentan los promedios de edad materna en cada uno de los grupos a comparar.

Al comparar los promedios de edades maternas mediante un análisis de varianza a un factor: condición del RN: sano o malformado, o condición de la madre: diabética gestacional y diabética pregestacional, o madre no diabética, se demostró que estos promedios difirieron entre sí $(\mathrm{p}<0,001)$ en ambos casos, siendo mayores los promedios de edad materna en el caso de niños malformados y en el caso de madres diabéticas.

Tabla 4. Análisis de varianza del peso de nacimiento según dos factores: condición de la madre: diabética gestacional (D G), diabética pregestacional (D M) o no diabética (N D) y condición del RN : sano o malformado

\begin{tabular}{|lccrr|}
\hline Fuente de variación & Grados de libertad & Suma de cuadrados & F & p \\
\hline Modelo & 5 & 1033073,0 & 5,87 & $<0,0001$ \\
Condición del RN & 1 & 4643842,5 & 13,18 & $<0,0001$ \\
Condición de la madre & 2 & 3038799,9 & 4,13 & 0,0140 \\
Interacción & 2 & 358331,1 & 0,51 & 0,6020 \\
Error & 257 & 95812787,0 & & \\
Total & 267 & 104886833,5 & & \\
\hline
\end{tabular}

Tabla 5. Edad materna (en años) según la condición del RN : malformado o no malformado, y según la condición de la madre: diabética gestacional (D G), diabética pregestacional (D M ) o no diabética (N D )

\begin{tabular}{|c|c|c|c|c|c|c|c|c|c|}
\hline \multirow[t]{2}{*}{$\begin{array}{l}\text { Condición } \\
\text { de la madre }\end{array}$} & \multicolumn{3}{|c|}{ No malformado } & \multicolumn{3}{|c|}{$\begin{array}{c}\text { Condición del Recién Nacido } \\
\text { Malformado }\end{array}$} & \multicolumn{3}{|c|}{ Total } \\
\hline & $\overline{\mathrm{x}}$ & Desv. St & $\mathrm{n}$ & $\overline{\mathrm{x}}$ & Desv. ST & $\mathrm{n}$ & $\overline{\mathrm{x}}$ & Desv. St & $\mathrm{n}$ \\
\hline ND & 28,2 & 6,29 & 132 & 33,5 & 6,13 & 14 & 28,6 & 6,45 & 146 \\
\hline DG & 32,4 & 5,05 & 52 & 32,5 & 6,04 & 46 & 32,5 & 5,51 & 98 \\
\hline $\mathrm{DM}$ & 33,6 & 4,58 & 18 & 34,5 & 5,01 & 6 & 33,8 & 4,59 & 24 \\
\hline Total & 29,7 & 6,25 & 202 & 32,9 & 5,93 & 66 & 30,5 & 6,32 & 268 \\
\hline
\end{tabular}




\section{Tabla 6. Análisis de varianza de la edad materna según dos factores: condición de la madre diabética: gestacional (D G), diabética pregestacional (D M ) o no diabética (N D) y condición de RN : malformado o no malformado}

\begin{tabular}{|lccrr|}
\hline Fuente de variación & Grados de libertad & Suma de cuadrados & F & p \\
\hline Modelo & 5 & 1566,1 & 8,98 & $<0,0001$ \\
Condición del RN & 1 & 116,9 & 3,53 & 0,0680 \\
Condición de la madre & 2 & 166,0 & 83,00 & 0,0950 \\
Interacción & 2 & 235,2 & 3,372 & 0,0360 \\
Error & 257 & 9521,1 & 34,9 & \\
Total & 267 & 11605,3 & & \\
\hline
\end{tabular}

Al realizar un análisis de varianza a dos factores (Tabla 6), se demostró que existía un efecto estadísticamente significativo del modelo en el promedio de la edad materna, principalmente debido a la interacción entre ambos factores (malformación del $\mathrm{RN}$ y diabetes materna) $(\mathrm{p}=0,036)$, los cuales se potencian, siendo menos relevante el efecto aislado de cada factor.

En relación al tipo de diabetes de la madre, se demostró, mediante prueba de Tukey, que las madres no diabéticas tenían menor edad promedio que las madres diabéticas ( $\mathrm{p}<0,05$ ), no existiendo diferencias significativas entre el promedio de la edad materna con diabetes gestacional y diabetes mellitus pregestacional.

La distribución de los sexos de los recién nacidos fue estadísticamente diferente en hijos de madres diabéticas que no diabéticas $\left(\chi^{2}=4,85\right.$; $\mathrm{p}=0,028)$ : en las primeras $43,4 \%(53 / 69)$ fueron varones, mientras que en los hijos de madres no diabéticas el 56,8\% (88/67) fueron varones.

\section{DisCUSIÓN}

El primer hecho importante que se muestra en este estudio, es que no hay diferencia significativa en la prevalencia al nacimiento de malformaciones congénitas en los hijos de madres con alguno de los dos tipos de diabetes estudiados, gestacional y pregestacional $\left(\chi^{2}=0,47 ; p<p=0,49\right)$. Adicionalmente, se demuestra que en ellos, en conjunto o por separado, la frecuencia de malformaciones congénitas es significativamente mayor que en los hijos de madres no diabéticas ( $p<0,00001$ ).

La frecuencia encontrada de madres diabéticas es igual a la comunicada por otros autores: 1,8\% para las mujeres diabéticas gestacionales y $0,3 \%$ para las pregestacionales.

En la Introducción dijimos que la tasa de prevalencia al nacimiento de MC entregadas por ECLAMC-Chile es de alrededor de 3\%, cifra que es mucho menor que la encontrada por nosotros en esta muestra $(8,14 \%)$, para el total de nacimientos del período. Este hecho no es nuevo, ya que siempre hemos encontrado una frecuencia de anomalías congénitas superior en el HCUCH que en el resto de los hospitales del ECLAMC, seguramente, por ser un hospital de referencia para este tipo de patologías ${ }^{7-9}$.

$\mathrm{Al}$ comparar las frecuencias de $\mathrm{RN}$ malformados entre el grupo de madres con DG y las DM, se comprueba que no hay diferencias significativas entre ambas $\left(\chi^{2}=0,47 ; \mathrm{p}=0,49\right)$, lo que podría explicarse por el pequeño tamaño muestral del segundo grupo. Pero la diferencia es altamente significativa, tanto en las madres con DG, como las con DM, cuando las comparamos con las madres no diabéticas.

Al revisar las malformaciones según su severidad o gravedad, se destaca que en los hijos de madres con DM son mucho más frecuentes las malformaciones mayores $(5 / 6-83,3 \%)$ que en los hijos de madres con DG (32/59-54,2\%), diferencia que no alcanza la significación estadística $\left(\chi^{2}=0,47 ; \quad p=0,49\right)$, probablemente debido al $n$ 
pequeño de DM. En los hijos de madres no diabéticas encontramos $10 \mathrm{MC}$ mayores (58\%) y 7 menores $(41,2 \%)$. Estas diferencias tampoco alcanzan la significancia estadística.

Esto concuerda con lo que ha sido comunicado por otros autores, que encuentran un aumento específico de las malformaciones mayores entre los hijos de madres diabéticas ${ }^{4}$. La asociación de DM materna con anomalías congénitas es bien conocida. Los niños hijos de madres insulinodependientes tienen un riesgo aumentado para malformaciones congénitas, especialmente mayores, multiorgánicas. Se comprometen con mayor frecuencia los sistemas cardiovascular, SNC, genitourinario y esquelético. Los estudios también muestran que los hijos de mujeres con diabetes gestacional, especialmente aquellas con hiperglicemia en ayunas, tienden a tener tasas más altas de $\mathrm{MC}^{4}$. La presentación de un caso de secuencia de regresión caudal, sugiere que la DG puede ser responsable del desarrollo de casos severos de displasia caudal, al igual que la $\mathrm{DM}^{5}$. Según Schaefer ${ }^{4}$, las anomalías congénitas en mujeres con DG y diabetes tipo 2, afectan a los mismos órganos y sistemas que han sido descritos en mujeres con diabetes tipo 1. Encuentra, al igual que nosotros, que las cardiopatías congénitas son el grupo más frecuente $(37,6 \%)$, seguidas por las musculoesqueléticas $(14,7 \%)$, SNC $(9,8 \%)$ y anomalías que comprometen múltiples órganos y sistemas $(16 \%)^{4}$.

En nuestro material, al igual que lo que afirma Schaefer ${ }^{4}$, llama la atención la prevalencia alta de malformaciones cardíacas en los hijos de madres con diabetes gestacional y pregestacional; la mitad de los RN malformados hijos de mujeres con DM presentaban una cardiopatía congénita. Además, $17,4 \%$ de los hijos de madres con diabetes gestacional, también tenían una anomalía cardíaca (8/46). La prevalencia al nacimiento de defectos cardíacos en hijos de madres no diabéticas fue de 3,4\%. Esta mayor frecuencia de malformaciones cardíacas ha sido comunicada por otros autores, al igual que las esqueléticas.

El embarazo ha sido frecuentemente destacado por tener un efecto diabetógeno en el metabolismo normal de los carbohidratos, lo que se evidencia por hiperglicemia e hiperinsulinemia como respuesta a la alimentación. Ello sitúa a la diabetes gestacional en $2 \%$ a $3 \%$ de las mujeres y provoca un empeoramiento del estado metabólico en mujeres con diabetes mellitus insulinodependientes ${ }^{10}$.

Ya que la disponibilidad de nutrientes para el feto depende primariamente del estado metabólico materno, se cree que estas aberraciones del metabolismo energético pueden producir complicaciones perinatales, incluyendo la embriopatía diabética. En efecto, la frecuencia de anomalías congénitas está aumentada entre los hijos de las mujeres diabéticas. Son responsables de aproximadamente $40 \%$ de todas las muertes perinatales. Recientes evidencias sugieren que la normalización de los niveles de glucosa en la sangre, unida a estrategias programadas de control y manejo, pueden disminuir la frecuencia de malformaciones congénitas, tanto como una mejoría de los indicadores de bienestar materno y feta ${ }^{10}$.

Sin embargo, para producir un impacto en incidencia de malformaciones, la normalización de la glicemia debe comenzar en el período preconcepcional y continuarse durante el período de embriogénesis. El control preconcepcional y los regímenes estrictos de alimentación, siguen siendo el centro de los programas de control de las mujeres diabéticas ${ }^{10}$.

Con los conocimientos actuales sobre esta materia, se puede sugerir que la diabetes gestacional es una entidad muy heterogénea, en la que se pueden incluir, a lo menos, dos tipos de diabetes, diabetes no gestacionales, no diagnosticadas previamente al embarazo o recientemente diagnosticadas durante la gestación. De esta manera, es posible que el efecto teratogénico de la enfermedad esté relacionado con una diabetes mellitus latente o no diagnosticada ${ }^{11}$.

Farrel $^{12}$, sugiere que es más probable que aquellas mujeres que presentaron una diabetes gestacional y diabetes en el postparto inmediato, hayan tenido una diabetes tipo 2 pregestacional no reconocida $\mathrm{y}$, por lo tanto, representan el mismo riesgo de anomalías congénitas que las pacientes con diabetes mellitus tipo 1 ó 2 establecidas.

Como en la actualidad, no se puede diferenciar ambas situaciones, se debe considerar como de riesgo para malformaciones todos aquellos embarazos complicados con diabetes gestacional.

A la vista de estos resultados, se podría concluir que el riesgo de tener un hijo malforma- 
do es alto, tanto para las mujeres con diabetes pregestacional como para las que presentan diabetes gestacional. Este hecho nos parece muy

\section{REFERENCIAS}

1. Ramírez R, Nazer J. Recién nacido hijo de madre diabética. En Nazer J, Ramírez R ed Neonatología. Santiago. Editorial Universitaria. 2003; 387-98.

2. [Castilla EE, Orioli IM. ECLAMC: The Latin American Collaborative Study of Congenital Malformations. Community Genetics 7: 76-94, 2004.] www.Eclamcnet.net

3. Uvena-Calabrezze J, Catalano P. The infant of the woman with gestational diabetes mellitus. Clin Obstet Gynecol 2000; 43: 127-39

4. Schaefer UM, Buchanan TA, Xiang AN, Peters RK, KIOS SL. Clinical predictors for a high risk development of diabetes mellitus in the early puerperium in women with recent gestational diabetes mellitus. Am J Obstet \& Gynecol 2002; 186: 751-6.

5. Versiani BR, Gilbert-Barnes E, Giuliono lR, Peres LC, Pina-Neto M. Caudal dysplasia secuence. Severe phenoty presenting in offspring of patients with gestacional and pregestational diabetes. Clinical Dismorphology 2004; 13: 1-5. importante, ya que es necesario conocer estos riesgos y los factores que contribuyen a aumentarlo, como la obesidad de la madre.

6. Shefield J, Buttler E, Casey B, McIntire D. Diabetes mellitus and infants malformations. Obstet \& Gynecol 2002; 100: 925-30.

7. Nazer J. En Neonatología. Nazer J, Ramírez R eds. 2003. Ed Universitaria. Santiago. Chile. Malformaciones congénitas p 433.

8. NAZER J. Anomalías congénitas estructurales en el recién nacido. Rev Hospital Clínico Universidad de Chile 2002; 13: 294-305.

9. Nazer J, ANTolini M, Jú́rez ME, Cifuentes L, Hübner ME, PaRdo RA y cols. Prevalencia al nacimientos de aberraciones cromosómicas en el Hospital Clínico Universidad de Chile. Período 1990-2001. Rev Méd Chile 2003; 131: 651-8.

10. Martínez-Frías ML, Bermejo E, Rodríguez-Pinilla E, PRIETO L, FríAs JL. Epidemiological analysis of outcomes of pregnancy in gestational diabetic mothers. Am J Obstet \& Gynecol 1998; 78: 140-5.

11. Farrel T, Neale L, Cundy T. Congenital anomalies in the offspring of women with type 2 and gestacional diabetes. Diabet Med 2002; 19: 322-6. 